\title{
GRB 091127/SN2009nz and the VLT/X-shooter spectroscopy of its host galaxy: probing the faint end of the mass-metallicity relation ${ }^{\star}$
}

S. D. Vergani ${ }^{1,2,3}$, H. Flores ${ }^{2}$, S. Covino ${ }^{1}$, D. Fugazza ${ }^{1}$, J. Gorosabel ${ }^{4}$, A. J. Levan ${ }^{5}$, M. Puech ${ }^{2}$, R. Salvaterra ${ }^{6}$, J. C. Tello ${ }^{4}$, A. de Ugarte Postigo ${ }^{7}$, P. D’Avanzo ${ }^{1}$, V. D’Elia ${ }^{8,9}$, M. Fernández ${ }^{4}$, J. P. U. Fynbo ${ }^{7}$, G. Ghirlanda ${ }^{1}$, M. Jelínek ${ }^{4}$, A. Lundgren ${ }^{10,11}$, D. Malesani ${ }^{7}$, E. Palazzi ${ }^{12}$, S. Piranomonte ${ }^{8}$, M. Rodrigues ${ }^{10}$, R. Sánchez-Ramírez ${ }^{4}$, V. Terrón ${ }^{4}$, C. C. Thöne ${ }^{4}$, L. A. Antonelli ${ }^{8}$, S. Campana ${ }^{1}$, A. J. Castro-Tirado ${ }^{4}$, P. Goldoni ${ }^{3,13}$, F. Hammer ${ }^{2}$, J. Hjorth ${ }^{7}$, P. Jakobsson ${ }^{14}$, L. Kaper ${ }^{15}$, A. Melandri ${ }^{1}$, B. Milvang-Jensen ${ }^{7}$, J. Sollerman ${ }^{16}$, G. Tagliaferri ${ }^{1}$, N. R. Tanvir ${ }^{17}$, K. Wiersema ${ }^{17}$, and R. A. M. J. Wijers ${ }^{15}$

1 INAF, Osservatorio Astronomico di Brera, via E. Bianchi 46, 23807 Merate, Italy e-mail: susanna.vergani@brera.inaf.it

2 GEPI-Observatoire de Paris Meudon, 5 place Jules Jansen, 92195 Meudon, France

3 Laboratoire Astroparticule et Cosmologie, 10 rue A. Domon et L. Duquet, 75205 Paris Cedex 13, France

${ }^{4}$ Instituto de Astrofísica de Andalucía (IAA-CSIC), Glorieta de la Astronomía s/n, 18008 Granada, Spain

5 Department of Physics, University of Warwick, Coventry, CV4 7AL, UK

${ }^{6}$ Dipartimento di Fisica e Matematica, Università dell'Insubria, via Valleggio 7, 22100 Como, Italy

7 Dark Cosmology Centre, Niels Bohr Institute, University of Copenhagen, Juliane Maries Vej 30, 2100 Copenhagen $\varnothing$, Denmark

8 INAF, Osservatorio Astronomico di Roma, via di Frascati 33, 00040 Monte Porzio Catone, Rome, Italy

9 ASI-Science Data Center, via Galileo Galilei, 00044 Frascati, Italy

10 European Southern Observatory, Alonso de Córdova 3107, Vitacura, Casilla 19001, Santiago 19, Chile

11 Joint ALMA Observatory, Alonso de Córdova 3107, Vitacura-Santiago, Chile

12 INAF, IASF di Bologna, via Gobetti 101, 40129 Bologna, Italy

13 Service d'Astrophysique, DSM/IRFU/SAp, CEA-Saclay, 91191 Gif-sur-Yvette, France

${ }_{14}$ Centre for Astrophysics and Cosmology, Science Institute, University of Iceland, Dunhagi 5, 107 Reykjavík, Iceland

15 Astronomical Institute Anton Pannekoek, University of Amsterdam, Science Park 904, 1098 XH Amsterdam, The Netherlands

16 The Oskar Klein Centre, Department of Astronomy, AlbaNova, Stockholm University, 10691 Stockholm, Sweden

17 Department of Physics and Astronomy, University of Leicester, University Road, Leicester LE1 7RH, UK

Received 19 July 2011 / Accepted 14 September 2011

\section{ABSTRACT}

\begin{abstract}
We perform a detailed study of the gamma-ray burst GRB 091127/SN 2009nz host galaxy at $z=0.490$ using the VLT/X-shooter spectrograph in slit and integral-field unit (IFU) mode. From the analysis of the optical and X-ray afterglow data obtained from ground-based telescopes and Swift-XRT, we confirm the presence of a bump associated with SN2009nz and find evidence of a possible jet break in the afterglow lightcurve. The X-shooter afterglow spectra reveal several emission lines from the underlying host, from which we derive its integrated properties. These properties agree with those of previously studied GRB-SN hosts and, more generally, with those of the long GRB host population. We use the Hubble Space Telescope and ground-based images of the host to determine its stellar mass $\left(M_{\star}\right)$. Our results extend to lower $M_{\star}$ values the $M$ - $Z$ plot derived for the sample of long GRB hosts at $0.3<z<1.0$ adding new information to probe the faint end of the $M-Z$ relation and the shift of the LGRB host $M-Z$ relation from that found from emission-line galaxy surveys. Thanks to the IFU spectroscopy, we can build the two-dimensional (2D) velocity, velocity dispersion, and star formation rate (SFR) maps. They show that the host galaxy has perturbed rotation kinematics with evidence of a SFR enhancement consistent with the afterglow position.
\end{abstract}

Key words. gamma-ray burst: individual: GRB 091127 - supernovae: individual: SN 2009nz - galaxies: ISM - galaxies: evolution

\section{Introduction}

The association of long gamma-ray bursts (LGRBs) with broadlined type Ic supernovae ( $\mathrm{SNe})$ is now well-established (e.g. Hjorth \& Bloom 2011 and references therein; see however GRB 060614: Della Valle et al. 2006; Fynbo et al. 2006; Gal-Yam et al. 2006). The spectral features of SNe have been

* Based on observations made with ESO Telescopes at Paranal Observatory under programmes ID 084.A-0260 and 086.A-0874. found in the afterglow spectra of seven LGRBs (see Starling et al. 2011 and references therein; Sparre et al. 2011; and for SN 2009nz Berger et al. 2011). The association with a SN can also be inferred from the rebrightening of the GRB afterglow optical lightcurve after a few days from the burst. The first pieces of evidence of these bumps were reported by Bloom et al. (1999), Galama et al. (2000), and Lazzati et al. (2001), and the spectroscopic observations obtained for some of them confirmed the association (e.g., Della Valle et al. 2003). The possibility that a massive collapsing star might launch relativistic jets capable 
of powering a gamma-ray burst was predicted theoretically by Woosley (1993) with the so-called collapsar model (MacFadyen \& Woosley 1999). Additional evidence of the LGRB SN Ic connection comes from the study of Kelly et al. (2008) showing that $\mathrm{SNe}$ Ic and LGRBs are similarly distributed within their hosts.

The SNe Ic, in particular the highly energetic, broad-lined Ic $\mathrm{SNe}$, are likely produced by massive, rotating Wolf-Rayet (WR) progenitors (see Crowther 2007 for a review), making this kind of stars the most likely candidate LGRB progenitors (as single stars or in binary systems). WR spectral features have indeed been found in the spectra of some LGRB host galaxies (Hammer et al. 2006; Han et al. 2010), although the spatially resolved observations performed for one of these hosts (GRB 980425) showed that the WR region was located hundreds of parsec away from the LGRB site (Hammer et al. 2006). The WR wind signatures could also be found in the afterglow spectra (van Marle et al. 2008), even if to date their claimed detections remain disputed (Schaefer et al. 2003; Mirabal et al. 2003; Fiore et al. 2005; Starling et al. 2005; Castro-Tirado et al. 2010; Chen et al. 2007).

The ratio of the LGRBs to $\mathrm{SN} \mathrm{Ib/c} \mathrm{rate} \mathrm{is} \mathrm{about} 1 \%$ (Guetta \& Della Valle 2007; Soderberg et al. 2010), hence only a very small fraction of massive stars dies producing a LGRB. It remains unclear what peculiar conditions lead a massive star to have the special kind of core collapse that triggers the formation of a jet and LGRBs. Metallicity is one of the fundamental parameters predicted to impact the evolution of massive stars as well as their explosive deaths and, together with rapid rotation, is expected to play a fundamental role in the formation of LGRBs (e.g., Heger et al. 2003; Yoon et al. 2010 and references therein). Modjaz et al. (2008) show that the SNe associated with LGRBs are more likely to exist in lower metallicity environments than broad-lined Ic SNe without a LGRB association.

A possible way to retrieve information on LGRB progenitors and the physical properties of LGRB regions is based on spectroscopy of their host galaxies. The global properties of the hosts (metallicity, star formation rate, etc.) can be determined and more detailed investigations can be performed for nearby galaxies for which, using multi-slit and/or integral field unit (IFU) spectroscopy, we can compile metallicity, SFR, density, and velocity maps. It is therefore possible to search for the peculiarities of the LGRB and its near-by regions and study the kinematics of the gas in the galaxy. To date, spatially resolved studies of LGRB host galaxies have been performed only for the low-redshift GRB 980425, GRB 060505, and GRB100316D ( $z=0.0085,0.0889,0.0593$, respectively; Hammer et al. 2006; Christensen et al. 2008; Thöne et al. 2008; Levesque et al. 2011).

The study of LGRB hosts can also provide useful constraints on galaxy evolution studies. The host galaxies of LGRBs are not selected based on luminosity (although the possibility of studying their properties is of course observationally partially biased towards brighter hosts), hence their study can be complementary to those of current surveys of galaxies. It has been shown that they are systematically offset to lower metallicities with respect to the mass-metallicity relation found from the surveys of emission-line galaxies (Han et al. 2010; Levesque et al. 2010; Mannucci et al. 2011). To explain this behaviour and build a complete picture of galaxy evolution, it is important to increase the size of the LGRB host sample to confirm this result and to determine whether and how these galaxies evolve at higher redshift. The IFU surveys of intermediate redshift galaxies performed in the past (e.g. Flores et al. 2006; Puech et al. 2006) demonstrated the power of this technique in helping to determine the dynamical properties of the galaxies, uncovering several galaxies with perturbed kinematics caused by either mergers or outflows, and therefore adding important pieces of information to the galaxy evolution scenario. With IFU observations of LGRB host galaxies, it will be possible to extend these studies to lower mass and lower metallicity objects.

Together with the analysis of the afterglow lightcurve, we present in this paper a detailed study of the host galaxy of GRB 091127 at $z=0.49044$ associated with SN 2009nz (Cobb et al. 2010; Berger et al. 2011), carried out using VLT/X-shooter (D'Odorico et al. 2004) echelle slit and IFU spectroscopy. This is the first paper presenting data obtained in the $\mathrm{X}$-shooter IFU mode.

In Sect. 2, we present the multiwavelength photometry of the GRB 091127 afterglow and host galaxy obtained with groundbased facilities and the Hubble Space Telescope (HST). These data and the Swift-XRT ones are fitted together to analyse the lightcurve (Sect. 3). Sections 4 and 5 are dedicated to the longslit and IFU spectroscopic data, respectively. The results on the integrated properties of the host galaxy are reported in Sect. 6, whereas in Sect. 7 the IFU 2D host galaxy maps are shown. Our findings are discussed in Sect. 8 and conclusions are drawn in Sect. 9.

\section{Photometry of the afterglow and of the host galaxy}

We obtained multiwavelength photometry of the afterglow lightcurve and the burst host galaxy using data acquired from $\sim 1$ h to $\sim 400$ days (observer frame) after the GRB explosion, using the HST and several ground based facilities (see Table 1; Fig. 1).

The early epoch $J$ and $H$ images, observed with REM-REMIR $^{1}$, were automatically reduced by the task PREPROCESS (di Paola 2001; sky subtraction, average of five dithered images). The photometry was performed with GAIA ${ }^{2}$ using an aperture of $5^{\prime \prime}$ ( 5 pixel) compatible with the measured FWHM (2.5"). The zero-point calibration is based on three 2MASS stars (Skrutskie et al. 2006) that are sufficiently bright in the GRB field. The TNG-DOLoRes ${ }^{3}$ images, 16 images in the $R$ filter for the afterglow observation, 2 images in the filters $B, V$, and $I$ for the late-time host observation, were shifted and averaged. The fringes in the $I$ images were removed using the frame of reference provided by the TNG web pages. The photometry was performed with GAIA using the PSF method (the measured seeing is $\sim 1^{\prime \prime}$ in all cases). The calibration of the zero point was based on nine secondary standard stars in the GRB field.

The reduction of both the optical and near-IR GTC ${ }^{4}, \mathrm{NOT}^{5}$, and $\mathrm{CAHA}^{6}$ data was done with $\mathrm{IRAF}^{7}$ following standard procedures. The photometry of the reduced images was carried out running PHOT aperture photometry as implemented in IRAF. We used as a radius of the aperture the FWHM of the seeing. The optical calibration was obtained observing a standard star at an airmass similar to the one of the GRB and assuming the extinction coefficients of La Palma. The $K$-band calibration was

\footnotetext{
1 http://www.rem.inaf.it/

2 http://astro.dur.ac.uk/ pdraper/gaia/gaia.html

3 http://www.tng.iac.es/

4 http://www.gtc.iac.es/en/pages/gtc.php

5 http://www.not.iac.es/

6 http://www. caha.es/

IRAF is a software distributed by National Optical Astronomy Observatories.
} 
Table 1. GRB 091127 OT and host galaxy photometry.

\begin{tabular}{|c|c|c|c|c|}
\hline Telescope & $\begin{array}{l}\text { Obs. time } \\
\text { (days) }\end{array}$ & Filter & $\operatorname{Mag}^{a}$ & $\begin{array}{l}\text { Exp. } \\
\text { time }(\mathrm{s})\end{array}$ \\
\hline TNG & 368.946 & $B$ & $24.25 \pm 0.18$ & 1200 \\
\hline GTC & 8.94568 & $g$ & $23.03 \pm 0.10$ & 90 \\
\hline GTC & 34.9434 & $g$ & $23.38 \pm 0.38$ & 30 \\
\hline GTC & 256.219 & $g$ & $24.23 \pm 0.26$ & 840 \\
\hline NOT & 48.8811 & $\stackrel{\circ}{V}$ & $23.43 \pm 0.05$ & 3200 \\
\hline TNG & 368.963 & $V$ & $23.73 \pm 0.18$ & 1200 \\
\hline TNG & 5.95030 & $R$ & $21.75 \pm 0.11$ & 960 \\
\hline OSN & 6.95917 & $R$ & $21.94 \pm 0.18$ & 1800 \\
\hline GTC & 8.94099 & $r$ & $22.21 \pm 0.06$ & 90 \\
\hline GTC & 34.9392 & $r$ & $22.52 \pm 0.11$ & 30 \\
\hline GTC & 256.230 & $r$ & $23.15 \pm 0.13$ & 600 \\
\hline $1.23 \mathrm{~m} \mathrm{CAHA}$ & 0.03599 & $I$ & $15.28 \pm 0.35$ & 120 \\
\hline $1.23 \mathrm{~m} \mathrm{CAHA}$ & 0.06309 & $I$ & $16.40 \pm 0.05$ & 120 \\
\hline NOT & 48.8440 & $I$ & $22.51 \pm 0.08$ & 720 \\
\hline TNG & 368.978 & $I$ & $22.77 \pm 0.19$ & 900 \\
\hline GTC & 8.95059 & $i$ & $22.00 \pm 0.07$ & 90 \\
\hline GTC & 34.9407 & $i$ & $22.03 \pm 0.06$ & 30 \\
\hline GTC & 256.241 & $i$ & $22.86 \pm 0.11$ & 840 \\
\hline GTC & 8.95529 & $z$ & $22.17 \pm 0.11$ & 90 \\
\hline GTC & 34.9767 & $z$ & $21.97 \pm 0.08$ & 30 \\
\hline GTC & 256.254 & $z$ & $22.39 \pm 0.28$ & 810 \\
\hline REM & 0.05176 & $J$ & $15.23 \pm 0.18$ & 150 \\
\hline REM & 0.06481 & $J$ & $15.31 \pm 0.14$ & 300 \\
\hline REM & 0.04730 & $H$ & $15.15 \pm 0.18$ & 150 \\
\hline REM & 0.05692 & $H$ & $14.90 \pm 0.11$ & 300 \\
\hline HST & 383.154 & $H$ & $21.62 \pm 0.04$ & 906 \\
\hline $3.5 \mathrm{~m} \mathrm{CAHA}$ & 4.93481 & $K$ & $19.45 \pm 0.17$ & 1895.4 \\
\hline
\end{tabular}

Notes. ${ }^{(a)}$ Magnitudes are corrected for Galactic extinction of $E(B-V)=$ $0.038 \mathrm{mag}$ (Schlegel et al. 1998). $B, V, R, I, J, H, K$ are Vega magnitudes, whereas $g, r, i$ and $z$ are $\mathrm{AB}$ magnitudes.

based on 12 bright unsaturated field stars present in the 2MASS catalogue.

Continuum observations at $870 \mu \mathrm{m}$ were also carried out, using the LABOCA bolometer array, installed on the Atacama Pathfinder EXperiment $\left(\mathrm{APEX}^{8}\right)$ telescope, on 2009 November 28 and 29. The total on-source integration time of the two combined epochs was $6.4 \mathrm{~h}(2.9$ and $3.5 \mathrm{~h}$ in the first and second epoch, respectively). Data were reduced using the Crush 2 software ${ }^{9}$. We obtained $3 \sigma$ upper limits of $14.9 \mathrm{mJy}$ and $13.2 \mathrm{mJy}$ for each of the two epochs, respectively, and $9.6 \mathrm{mJy}$ for the combined epochs.

Host galaxy observations of GRB 091127 using the HST were obtained on 16 December 2010, utilizing the WFC3 in the $H$ band $(F 160 W)$. An exposure time of $906 \mathrm{~s}$ was obtained in two dithered exposures, and reduced in the standard HST fashion. At the location of the optical afterglow, we clearly see the underlying host galaxy, whose centroid is offset relative to the position of the optical afterglow by only $\sim 0.2^{\prime \prime}$, confirming the analysis of Cobb et al. (2010). The host has $H(\mathrm{AB})=22.88 \pm 0.04$, $H($ Vega $)=21.62 \pm 0.04$, which, considering the optical host galaxy magnitudes obtained with the TNG, suggests that, like many LGRB hosts the host of GRB 091127 is relatively blue.

We calculated the probability $P_{\mathrm{ch}}$ that the identified galaxy is a chance superposition and not physically related to the GRB.

\footnotetext{
8 APEX is a collaboration between the Max-Plank-Institut für Radioastronomie, the European Southern Observatory and the Onsala Space Observatory.

9 http://www. submm. caltech.edu/ sharc/crush/
}

Using the procedure described in Bloom et al. (2002), we find that $P_{\mathrm{ch}} \sim 4 \times 10^{-3}$.

\section{Analysis of the afterglow emission}

We added to the optical/near-infrared (NIR) observation presented in Sect. 2 the X-ray afterglow products (see Fig. 1) found in the Swift-XRT light-curve and spectral repositories (Evans et al. 2007, 2009).

To analyse the afterglow emission, we fitted the photometric data in Table 1 together with the data published by Cobb et al. (2010) and the X-ray data with power-laws both for the spectral and temporal regimes, following usual conventions $\left(F_{v} \propto t^{-\alpha} v^{-\beta}\right)$. For the optical spectral analysis, we also considered rest-frame extinction following the Milky Way (MW), Large and Small Magellanic Clouds (LMC and SMC), and starburst (SB) recipes (Pei 1992; Calzetti et al. 1994). For the X-ray data, we considered neutral rest-frame absorption (Morrison \& McCammon 1983). In both cases, the effects of extinction by dust and absorption by gas in the Galaxy had been previously removed. For the optical data fit, we used a broken power-law with the addition of a late-time constant component, the host galaxy, and a SN component modelled following SN 1998bw (Galama et al. 1998; McKenzie \& Schaefer 1999; Sollerman et al. 2000; Patat et al. 2001; Sollerman et al. 2002), i.e. the same as that used by Cobb et al. (2010). The SN template was $\mathrm{K}$-corrected assuming a linear relation between the adjacent bands of the SN 1998bw observations. A possible scaling factor $k$ for the whole SN component was also introduced into the fit. The temporal properties of the SN turned out to be fully consistent in all bands with SN 1998bw shifted to the redshift of GRB 091127 and with $k=1.1$. It dominates the optical lightcurve from about a week and is significant up to about 200 days from the burst, when the host galaxy stable emission began to be observed. Berger et al. (2011) claimed the detection of supernova features in their Gemini spectra, confirming the association of GRB 091127 with a SN and finding that the SN has spectral properties similar to those of XRF 060218/SN2006aj.

Although the global shape of the optical light-curve allowed us to clearly separate the various components, it immediately turned out that there are severe cross-calibration uncertainties among the many instruments providing the available data. Cobb et al. (2010) mention a systematic calibration error of 0.05 based on their data alone. To obtain an acceptable $\chi^{2}$ for the global fit, we needed to add a $10 \%$ uncertainty in quadrature. This effectively means that we are unable to study possible low-amplitude variations, and only the global light-curve shape can be effectively modelled.

The X-ray light-curve is remarkably smooth and could be accurately modelled by a broken power-law with a break at about $33 \mathrm{ks}$. The break is consistent with being simultaneous at optical wavelengths. The post-break decay slopes are consistent between optical and X-rays within the errors, while the pre-break decay in the optical is significantly shallower than at higher energies. The X-ray spectral slope is slightly lower than 1, while, within the weak constraints of the fit, the optical spectral slope is much harder and it shows no evidence of spectral evolution (see also Cobb et al. 2010). This is not unprecedented behaviour, as very blue optical spectra have been previously reported (e.g. for GRB 060908, Covino et al. 2010). The optical spectral slope and rest-frame extinction show a high degree of degeneracy. The best fit would indeed require a non-negligible rest-frame absorption following the LMC extinction curve (see Table 2). All other recipes provided poorer fits. Cobb et al. (2010) for 


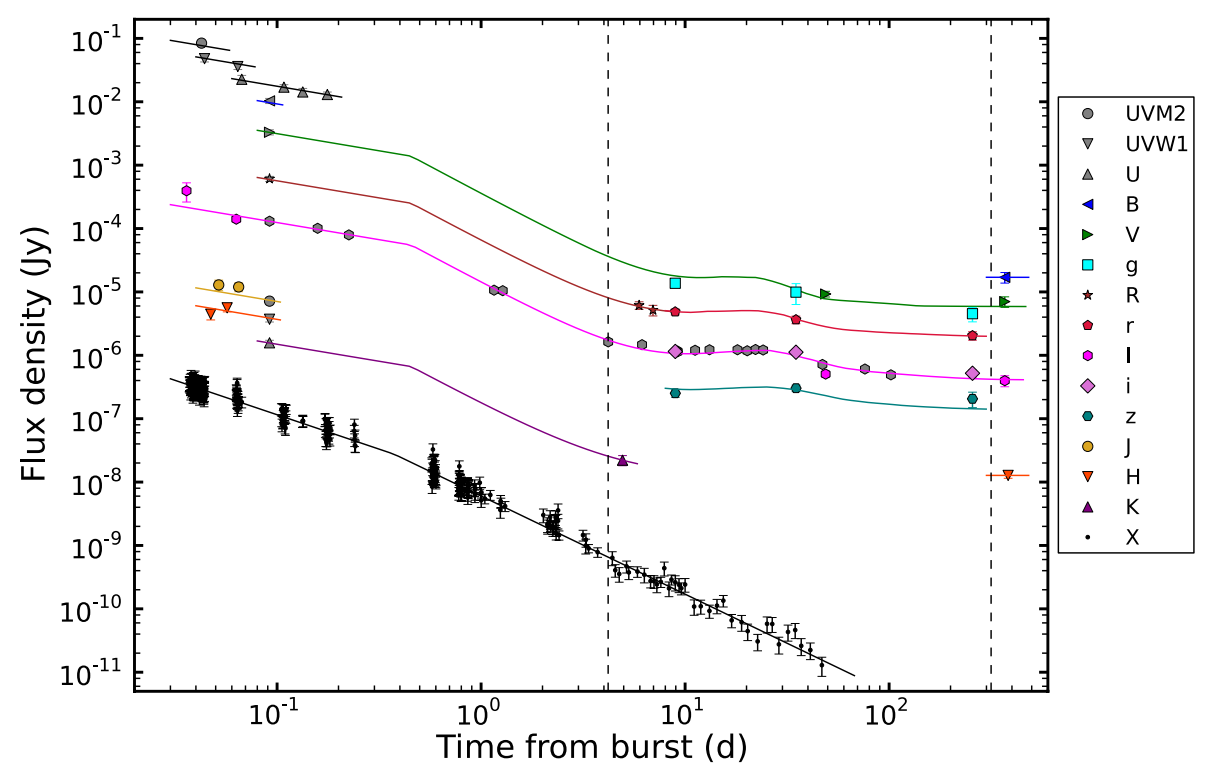

Fig. 1. Optical and X-ray light-curves of the GRB 091127 afterglow. The symbol legend is to the right of the plot. Fluxes have been rescaled for clarity, except for the $R$ - and $r$-filters $(U V M 2 \times 200, U V W 1 \times 100, U \times 50, B \times 20, V \times 6, g \times 6, I \times 0.2, i \times 0.2, z \times 0.05, J \times 0.01, H \times 0.005$, $K \times 0.002$, and $X \times 0.01$ ). Coloured symbols correspond to our data set, whereas grey symbols refers to the data published in the literature (see Cobb et al. 2010 and references therein). The solid curves show the best-fit relation for each band. A colour version of this figure is available online.

Table 2. Fit results.

\begin{tabular}{|c|c|c|}
\hline \multicolumn{3}{|c|}{ Optical data } \\
\hline & Best fit & Range \\
\hline$\alpha_{1}$ & 0.51 & {$[0.30,0.73]$} \\
\hline$\alpha_{2}$ & 1.74 & {$[1.46,2.08]$} \\
\hline$t_{\text {break }}$ (days) & 0.45 & {$[0.24,0.71]$} \\
\hline$\beta$ & +0.18 & {$[-0.43,0.81]$} \\
\hline$E(B-V)_{\mathrm{LMC}}$ & 0.16 & {$[0.03,0.31]$} \\
\hline$k$ & 1.1 & {$[0.71,1.48]$} \\
\hline$\chi^{2} /$ d.o.f. & \multicolumn{2}{|c|}{$53.96 / 44=1.23$} \\
\hline \multicolumn{3}{|c|}{ X-ray data: temporal analysis } \\
\hline & Best fit & Range \\
\hline$\alpha_{1}$ & 1.09 & {$[1.03,1.14]$} \\
\hline$\alpha_{2}$ & 1.55 & {$[1.51,1.58]$} \\
\hline$t_{\text {break }}$ (days) & 0.38 & {$[0.29,0.51]$} \\
\hline$\chi^{2} /$ d.o.f. & \multicolumn{2}{|c|}{$376.09 / 364=1.03$} \\
\hline \multicolumn{3}{|c|}{ X-ray data: spectral analysis } \\
\hline & Best fit & Range \\
\hline$N_{\mathrm{H}}\left(10^{21} \mathrm{~cm}^{-2}\right)$ & 1.31 & {$[0.83,1.81]$} \\
\hline$\beta$ & 0.93 & {$[0.82,1.04]$} \\
\hline C-stat(d.o.f.) & \multicolumn{2}{|c|}{$327.42(395)$} \\
\hline
\end{tabular}

Notes. Error ranges for the optical analysis and the X-ray temporal analysis are at $1 \sigma$, whereas for the X-ray spectral analysis they represent the $90 \%$ confidence level.

their observation covering a shorter wavelength range find that a smaller amount of absorption $\left(A_{\mathrm{V}}=0.2 \mathrm{mag}\right.$, corresponding to $E(B-V)_{\mathrm{LMC}}=0.06 \mathrm{mag}$ using their parameterization $)$ is compatible with their data.

The results of the fits are shown in Table 2.

\section{X-shooter long slit spectroscopy}

Our X-shooter long-slit spectra of GRB 091127 were obtained under the GTO program (084A-0260; PI: J. Fynbo) dedicated to GRB afterglow spectroscopy. For technical reasons, we could start the observation only about 4.2 days after the GRB explosion (Thöne et al. 2009), at 03:17 UT on Dec 2, 2009. The observation consisted of $4 \times 1500 \mathrm{~s}$ exposures in the UVB, VIS, and NIR arms (see Table 3) with an average seeing of $1^{\prime \prime}$.

We processed the frames using version 1.2.0 of the X-shooter data reduction pipeline (Goldoni et al. 2006). The data reduction was performed with the following steps. The raw frames were first subtracted and cosmic ray hits were detected and removed using the method developed by van Dokkum (2001). The frames were then divided by a master flat-field produced using daytime flat-field exposures with halogen lamps. The spectral orders were extracted and rectified in wavelength space using a wavelength solution previously obtained from calibration frames. The resulting rectified orders were then shifted and added to superimpose them thus obtaining the sky subtracted 2D spectrum. After these steps, the orders were merged and in the overlapping regions the merging was weighted by the errors that were propagated during the process. From the resulting 2D merged spectrum, a one dimensional spectrum was extracted at the source position with the corresponding error files and bad pixel maps.

The afterglow continuum is detected across the whole spectral range, even if its signal-to-noise ratio $(\mathrm{S} / \mathrm{N})$ is pretty low (also because of the effect of the nearby Moon) reaching a maximum of 5.5 in the VIS arm of the spectrum. Several emission lines from the host galaxy of GRB 091127 are clearly identified, which are superimposed on the afterglow continuum, in the UVB and VIS spectra at a common redshift of $0.49044 \pm 0.00008$ (see also Thöne et al. 2009; Cucchiara et al. 2009) and spanning about $150 \mathrm{~km} \mathrm{~s}^{-1}$. We do not detect any line in the NIR spectra. The overall one-dimensional (1D) spectrum was flux calibrated using the reference standard stars observed during the same night and was subsequently cross-checked and corrected using the corresponding magnitudes of the afterglow according to the photometric data and fit reported in Sect. 2. Slit-aperture flux loss corrections are therefore included in this procedure.

The observed emission-line fluxes (see Fig. 2) were measured using a self-developed code and cross-checked with the 
Table 3. X-shooter observation log.

\begin{tabular}{lccccccc}
\hline \hline GRB 091127 & $\begin{array}{c}\text { Date } \\
\text { Program ID }\end{array}$ & Mode & Arm & Binning & $\begin{array}{c}\text { Total exp. time } \\
\text { on source (s) }\end{array}$ & $\begin{array}{c}\text { Slit width } \\
\left({ }^{\prime \prime}\right)\end{array}$ & $\begin{array}{c}\text { Resolution } \\
\lambda / \Delta \lambda\end{array}$ \\
\hline slit & 2009 Dec. 02 & Nodding & UVB & $1 \times 2$ & 6000 & 1.0 & 5100 \\
& 084A-0260(C) & & VIS & $1 \times 2$ & 6000 & 0.9 & 8800 \\
& & & NIR & $1 \times 1$ & 6000 & 0.9 & 5100 \\
\hline IFU & 2010 Oct. 10 & Staring & UVB & $1 \times 1$ & 3300 & 0.6 & 7900 \\
& & & VIS & $1 \times 1$ & 3300 & 0.6 & 12600 \\
& & & NIR & $1 \times 1$ & 3300 & 0.6 & 8100 \\
\hline
\end{tabular}

Notes. From Col. 2 to 8, we report the observation date together with the observing run, the observation mode, the arms used, the corresponding binning, total exposure time, slit width, and central wavelength resolution, for both slit and IFU observations.

Table 4. Observed emission-line fluxes corrected for Galactic extinction.

\begin{tabular}{lc}
\hline \hline Emission line & $\begin{array}{c}\text { Flux } \\
10^{-17} \mathrm{erg} \mathrm{s}^{-1} \mathrm{~cm}^{-2}\end{array}$ \\
\hline$\left[\mathrm{O}_{\text {II }}\right] \lambda 3726$ & $5.0 \pm 1.2^{a}$ \\
{$\left[\mathrm{O}_{\text {II }}\right] \lambda 3729$} & $8.2 \pm 1.2^{a}$ \\
{$[\mathrm{Ne}$ III $] \lambda 3869$} & $<2.8$ \\
$\mathrm{H} \delta$ & $<2.5$ \\
$\mathrm{H} \gamma$ & $1.0 \pm 0.4$ \\
{$\left[\mathrm{O}_{\text {III }}\right] \lambda 4363$} & $<1.6$ \\
$\mathrm{H} \beta$ & $2.0 \pm 0.3$ \\
{$\left[\mathrm{O}_{\text {III }}\right] \lambda 4959$} & $2.8 \pm 0.3$ \\
{$\left[\mathrm{O}_{\text {III }}\right] \lambda 5007$} & $7.9 \pm 0.3$ \\
$\mathrm{H} \alpha$ & $5.5 \pm 0.3$ \\
{$[\mathrm{~N}$} & $<1.0$ \\
{$\left[\mathrm{~S}_{\mathrm{II}}\right] \lambda 676717$} & $1.5 \pm 0.5^{b}$ \\
{$\left[\mathrm{~S}_{\text {II }}\right] \lambda 6731$} & $<1.2$ \\
\hline
\end{tabular}

Notes. Upper limits are calculated at 3- $\sigma$. Errors shown here do not take into account a $10 \%$ systematic error caused by flux calibration uncertainties. ${ }^{(a)}$ The larger errors in the $\left[\mathrm{O}_{\text {III }}\right]$ fluxes are due to the nearby Moon and to the $\left[\mathrm{O}_{\mathrm{II}}\right]$ lines falling right in the range of the dichroic between the UVB and VIS arm where the transmission is therefore not optimal. ${ }^{(b)}$ Tentative detection: possible emission near a sky line.

SPLOT task in IRAF. The results, corrected for Galactic extinction, are listed in Table 4.

We searched for absorption lines but we can only report a tentative $\mathrm{Mg}_{\text {II }} \lambda 2796$ absorption at the limit of the detection, with a rest-frame equivalent width of about $2.5 \AA$ blueshifted by about $460 \mathrm{~km} \mathrm{~s}^{-1}$ from the emission lines.

\section{X-shooter IFU spectroscopy}

The X-shooter IFU spectra of GRB 091127 were obtained under excellent seeing conditions in October 2010 within the ItalianFrench GTO program (086A-0874; PI: S. Piranomonte) dedicated to GRB host galaxy spectroscopy (see Table 3). We used an object-sky sequence with an exposure time of $3300 \mathrm{~s}$ in each position. The position of the IFU field of view is shown in Fig. 3. The IFU was positioned on the host galaxy centre using blind offsets. The pointing error should be smaller than $0.1^{\prime \prime}$.

\subsection{IFU data reduction}

The IFU data reduction recipe remains under development within the ESO pipeline. We were able however to reduce the VIS arm data, using the recipe developed for the slit observations and correcting for the sky background via standard programs under IRAF and IDL. In a different way from the slit mode, for the IFU mode the atmospheric dispersion compensators (ADCs) are not in the optical path, hence the IFU mode is strongly affected by the atmospheric dispersion. Specific IDL programs were used to correct the atmospheric dispersion and construct the datacube (see Flores et al. 2011). The standard star GD71 was used to calibrate the flux of the datacube.

The final datacube (constructed around the $[\mathrm{O}$ III $] \lambda 5007$ emission line) has a spatial resolution of $0.1 \times 0 . ' 6$ (equivalent to $0.6 \times 3.6 \mathrm{kpc}^{2}$ ) and we kept the spectral resolution of the observations $(R=12600$ for the VIS arm).

\section{Integrated properties of the host galaxy}

Using the emission lines present in the slit spectrum, we can determine some integrated properties of the host galaxy. Assuming a temperature $T_{\mathrm{e}}=10000 \mathrm{~K}$, from the $\left[\mathrm{O}_{\text {II }}\right] \lambda \lambda 3727,3729$ and $\left[\mathrm{S}_{\mathrm{II}}\right] \lambda 6717,6731$ ratio, we infer an electron density $n_{\mathrm{e}} \leq$ $200 \mathrm{~cm}^{-3}$. The comparison of the observed flux ratio of the Balmer hydrogen emission lines with the values expected in the case B recombination in Osterbrock (1989) shows that no significant Balmer absorption correction is needed and that the ratios are consistent with $A_{\mathrm{V}}=0 \mathrm{mag}$ (within the errors, values of $A_{\mathrm{V}}<0.3$ mag are allowed), indicating that the host galaxy is blue and dominated by a young star population as also suggested by the galaxy colours.

We determine the metallicity of the host using the $R_{23}$ calibrator of the Kobulnicky \& Kewley (2004) method as described in Kewley \& Ellison (2008). We find that $12+\log (\mathrm{O} / \mathrm{H})=$ $8.72 \pm 0.16$ using the lower branch formula and $12+\log (\mathrm{O} / \mathrm{H})=$ $8.04 \pm 0.16$ for the upper branch. This indicates that we are at the turnover point of the double-valued $R_{23}$ diagnostic, which in fact occurs at the maximum value of $\log \left(R_{23}\right)=1$ (see Figs. 6 and 7 of Kobulnicky \& Kewley 2004) corresponding to the value determined from our data. From the models, the metallicity corresponding to this value is $12+\log (\mathrm{O} / \mathrm{H}) \sim 8.4$ (Kobulnicky \& Kewley 2004), with an error of $\sim 0.1$ dex.

Using the relations found by Kewley \& Ellison (2008), we can convert this value to the Pettini \& Pagel (2004) O3N2 diagnostic finding that $12+\log (\mathrm{O} / \mathrm{H})=8.2$. This result is consistent with the limit on the metallicity determined using the $\mathrm{O} 3 \mathrm{~N} 2 \mathrm{di}-$ agnostic with our data, i.e. $12+\log (\mathrm{O} / \mathrm{H})<8.3$. We note that this diagnostic is valid only for $\mathrm{O} 3 \mathrm{~N} 2<2$. We can only assume that this condition is satisfied for the GRB 091127 host, since we have only an upper limit for the [N II] flux giving O3N2 > 1.2.

We also determine the metallicity using the method followed by Mannucci et al. (2010) based on the simultaneous fitting of strong line ratios described in Maiolino et al. (2008). We find in this case that $12+\log (\mathrm{O} / \mathrm{H})=8.34_{-0.20}^{+0.10}$. 

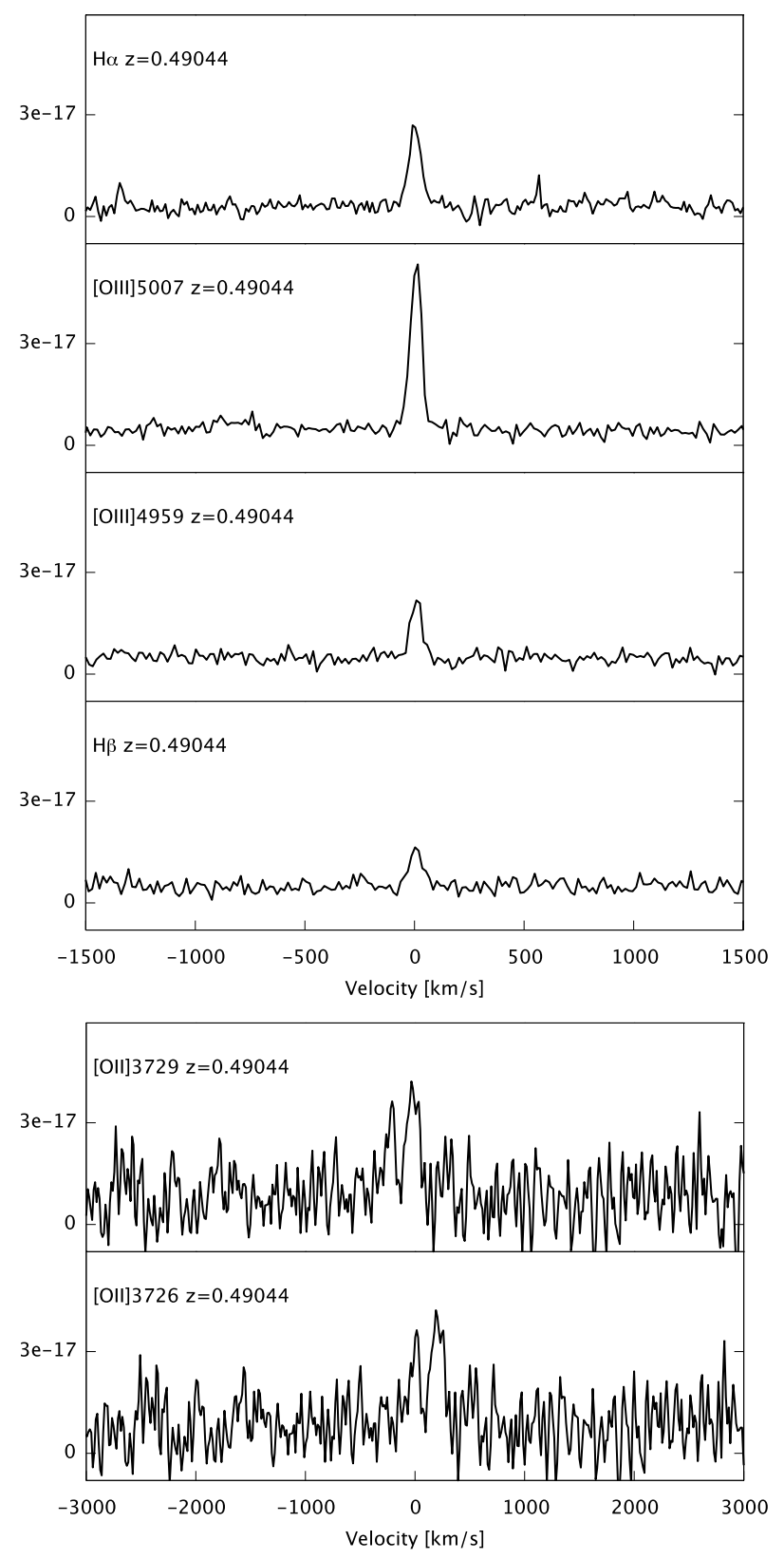

Fig. 2. Emission lines in the 1D slit VIS and UVB spectra (upper and lower panel, respectively), shown in the velocity space centered at $z=$ 0.49044. Flux units are $\operatorname{erg~s}^{-1} \mathrm{~cm}^{-2} \AA^{-1}$.

We calculate the SFR using the $\mathrm{H} \alpha$ emission line following the prescription given in Kennicutt (1998) and considering both the initial mass function (IMF) of Salpeter (1955) and that proposed by Baldry \& Glazebrook (2003) used by Savaglio et al. (2009) for GRB host galaxies. We find $S F R_{\mathrm{H} \alpha}=0.40,0.22 \pm$ $0.03 M_{\odot} \mathrm{yr}^{-1}$, respectively.

We use the HST and TNG late-time photometry of the GRB host to build the SED and infer the stellar mass $\left(M_{\star}\right)$ of the host galaxy. We fit the SED using Charlot \& Bruzual models (Bruzual \& Charlot 2003; Bruzual 2007). We assume a Salpeter IMF and constructed grids of $\tau$-exponentially declining star formation histories spaced at $0.1 \mathrm{Gyr}$ intervals from $\tau=0$ to $0.5 \mathrm{Gyr}$, and at $0.5 \mathrm{Gyr}$ intervals beyond, and age $t$. Following the results obtained from the slit spectroscopy, we fix $Z=0.008$ (that, among the values available, is the closest to the $12+\log (\mathrm{O} / \mathrm{H})$ metallicity determined above) and

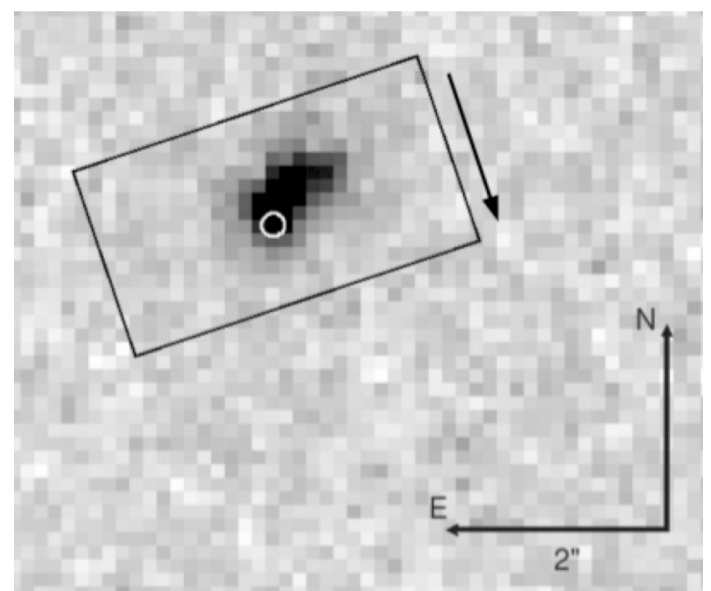

Fig. 3. HST $H$-band (F160W) image of the field of GRB 091127. The 1". $8 \times 3$ ". 5 IFU field of view is shown over the GRB host galaxy. The black arrow represents the direction of the IFU maps. The afterglow position (see Sect. 7.1) is indicated by the white circle.

$A_{\mathrm{V}}=0$ mag. Within a confidence interval of $68 \%$, we find that $\tau \in[100 ; 6000] \mathrm{Myr}, t \in[227 ; 905] \mathrm{Myr}$, and the stellar mass is $\log \left(M_{\star} / M_{\odot}\right)=8.6 \pm 0.1$. We consider the average conversion factor for deriving stellar masses using the IMF adopted in Savaglio et al. (2009), and obtain a mass corresponding to $\log \left(M_{\star} / M_{\odot}\right)=8.3$. Levesque et al. (2010) found that their masses determined using a Chabrier (2003) IMF are consistent with those of Savaglio et al. (2009), hence this latter value can be used when comparing with the masses of the GRB host galaxies studied by Levesque et al. (2010).

The specific star formation rate (SSFR) calculated with the SFR and $M_{\star}$ determined above is $\log (S S F R)\left(\mathrm{yr}^{-1}\right)=-9.0 \pm 0.1$.

From the host-galaxy late-time photometry, we derive an absolute $B$-band magnitude of $M_{B}=-18.4$. The host galaxy is therefore sub-luminous even considering only the luminosity function of irregular and starburst galaxies (Dahlen et al. 2005).

\section{IFU 2D maps}

\subsection{Velocity and velocity dispersion maps}

We used the software developed for the FLAMES/GIRAFFE instrument to recover the velocity field and the velocity dispersion map (Flores et al. 2006; Yang et al. 2008). Velocity fields were derived after subtraction of a $\sigma$-clipped mean of all velocities, and considering only spaxels with $S / N>4$. The fit to each accepted spaxel was checked by eye.

Figure 4 shows the velocity field and the velocity dispersion $(\sigma)$ maps obtained using the [O III] $\lambda 5007 \AA$ emission line of the host galaxy. For clearer viewing, we show the interpolated maps (spaxels of 0 . $^{\prime} 1 \times 0 .{ }^{\prime} 1$, real spaxels being $0{ }^{\prime} 1 \times 00^{\prime} 6$ ). The velocity map indicates a rotating galaxy and the $\sigma$-map shows an offset of the velocity dispersion peak from the galaxy center. These kinematics were defined as perturbed rotation by Flores et al. (2006) for their 3D VLT/GIRAFFE galaxy survey. Even using the HST image of the galaxy, it is difficult to check whether the morphology and the kinematics have the same principal axis, preventing a deeper interpretation of both maps. We can only discern that the velocity field does not seem to follow the galaxy major-axis direction. Galaxies for which the velocity field was not aligned with their major axis were found in the 3D VLT/GIRAFFE survey and defined as galaxies with complex kinematics (e.g., Flores et al. 2006; Puech et al. 2006). 


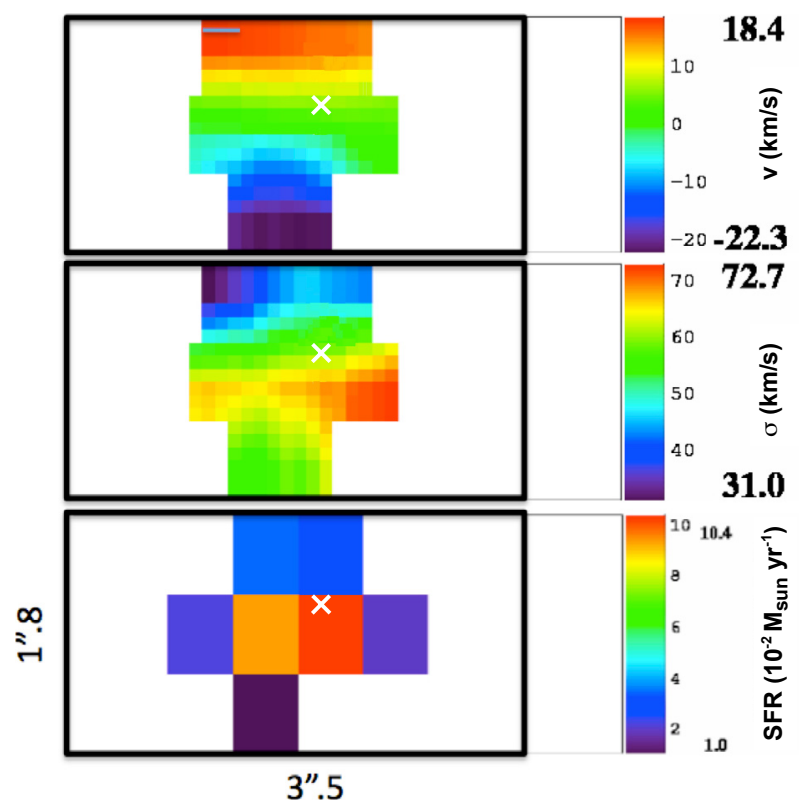

Fig. 4. From top to bottom: IFU velocity, velocity dispersion, and SFR (Salpeter IMF) maps $\left(1^{\prime \prime}=6.018 \mathrm{kpc}\right)$. Each map is orientated following the direction of the arrow reported in Fig. 3 (bottom to top). The afterglow position is indicated by the white cross. The velocity and velocity dispersion maps are interpolated to spaxels of 0 .' $1 \times 0$ '. 1 for clearer viewing. A colour version of this figure is available online.

Using the HST host galaxy image and the GEMINI afterglow images, we could determine an offset for the afterglow position of $0.29 \pm 0.05^{\prime \prime}$ south and $0.08 \pm 0.05^{\prime \prime}$ east from the host galaxy centre (we found comparable results also using different afterglow images from our dataset). While the offset in the southern direction is consistent with that found by Cobb et al. (2010), they found a small offset in the western direction. The discrepancy could be due to the higher resolution of the host galaxy of the HST image. In both cases, the GRB afterglow is located at the border of the high dispersion region.

\subsection{SFR map}

The SFR map was reconstructed from the $\mathrm{H} \alpha$ emission line assuming no extinction (as deduced from the slit observation). Given the weakness of the $\mathrm{H} \alpha$ emission line, we build a datacube with a spatial resolution of $0.5 \times 0.6$ (equivalent to $3.0 \times 3.6 \mathrm{kpc}^{2}$ ), to increase the $S / N$ of each spaxel. The emission of each spaxel was measured with automatic software and manually with IRAF. Only spaxels with a $S / N \geq 3$ are considered in the map shown in Fig. 4. The peak of the star formation is close to the position where the peak of $\sigma$ is detected. The GRB afterglow location is consistent with the star-formation peak region.

\section{Discussion}

\subsection{Jet break}

Although a full analysis of the temporal and spectral behaviour of the afterglow is beyond the purpose of this paper, it is interesting to discuss some of its main features. A break is observed simultaneously in the X-ray and optical light curves. This has been traditionally interpreted in terms of a jet outflow. In this case, according to the standard afterglow model (Zhang \& Mészáros 2004 and references therein), the post-break decay

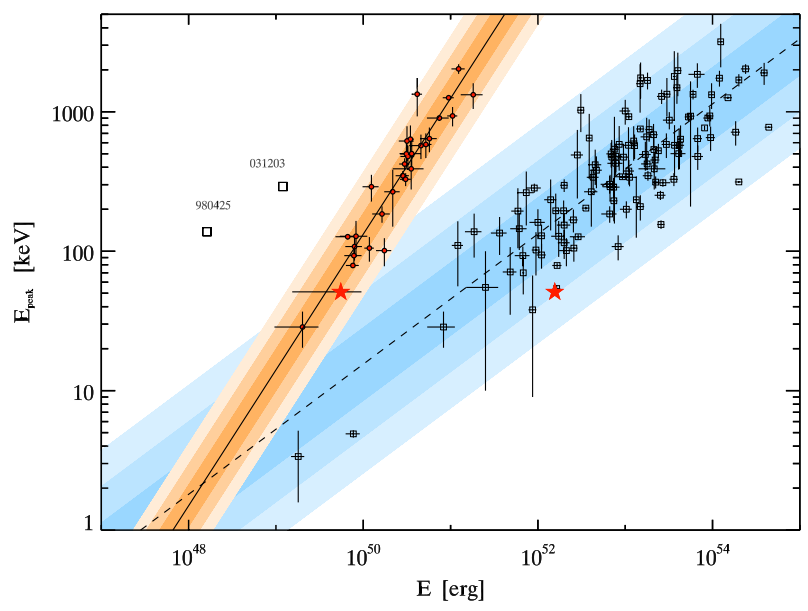

Fig. 5. $E_{\text {peak }}-E_{\text {iso }}$ (empty squares) and $E_{\text {peak }}-E_{\gamma}$ (red dots) correlations for GRBs. The shaded regions for the two correlations represent the $1,2,3 \sigma$ scatter in the data points (computed perpendicularly) with respect to the best-fit correlation (solid and dashed lines). The position of GRB 091127 in both correlations is shown by the red stars. A colour version of this figure is available online.

slope is independent of the wavelength and determined from the electron energy-distribution index.

For GRB 091127, the X-ray light curve is better sampled and not contaminated by the SN and host components. However, the X-ray decay after the break is too shallow even adopting an extreme value for the electron distribution index $p<1$ (formally $p \approx 0.2$ is required, an unphysical and unpredented value), which does not favour this model. The behaviour of the light curve after the jet break is however determined by the complex physics that regulate the jet sideway expansion, and is therefore quite uncertain (Granot 2007; Meliani \& Keppens 2010; van Eerten \& MacFadyen 2011). Limiting our analysis to before the jet break, our data are roughly consistent with model predictions. A flat electron index is required given the hard slope $\beta_{\mathrm{X}}$ of the X-ray spectrum $\left(p=2 \beta_{\mathrm{X}}=1.86 \pm 0.20\right)$. The optical and X-ray decay slopes predicted for this value of $p$ are $\alpha_{\text {opt }}=0.72 \pm 0.04, \alpha_{\mathrm{X}}=0.97 \pm 0.04$, which are compatible with the observed values within the errors (Table 2). A low value of $p$ also predicts a blue optical spectrum with $\beta_{\text {opt }}=0.43 \pm 0.10$, which is again consistent with the observed optical SED.

A different possible way to explain the shallow decay of the optical and X-ray light curves is the long-lasting energy injection in the fireball (e.g., Zhang et al. 2006), which has been invoked to explain several the X-ray light curves (e.g., Panaitescu et al. 2006; Liang et al. 2007). In this case, the break might either pinpoint the end of the energy injection, or represent the usual jet break, in which case energy injection would last until after the end of the Swift observations. The former possibility is ruled out because the observed $\alpha$ and $\beta$ in the X-ray range do not obey the expected closure relations (Zhang \& Mészáros 2004). Even if energy injection is significant, we therefore conclude that the most likely interpretation of the observed break is the jet effect.

This interpretation is consistent with the typical break times of cosmological GRBs (e.g., Ghirlanda et al. 2005). Using the Fermi-GBM spectral parameters reported by Nava et al. (2011) (in agreement with those of the Fermi team GCN; Wilson-Hodge \& Preece 2009), we show in Fig. 5 that GRB 091127 is consistent with the peak energy $\left(E_{\text {peak }}\right)$ - isotropic energy $\left(E_{\text {iso }}\right)$ correlation and the $E_{\text {peak }}-$ collimation correct energy $\left(E_{\gamma}\right)$ correlation (Amati 2006; Ghirlanda et al. 2004). 


\subsection{Properties of the GRB 091127 host}

The integrated properties of the host galaxy of GRB 091127 (low mass, low luminosity, low metallicity, and high SSFR) are comparable with those of the other host galaxies of LGRBs associated with SNe (Sollerman et al. 2005; Starling et al. 2011) and more in general with LGRB hosts (e.g. Savaglio et al. 2009; Levesque et al. 2010). Our flux measurements implies an $A_{\mathrm{V}}$ consistent with zero, in disagreement with the value obtained from the afterglow light-curve fitting. This indicates that the dust responsible for the GRB afterglow extinction is spatially localized and does not affect the overall emission of the galaxy.

Interest has increased in the study of the mass-metallicity $(M-Z)$ relation of LGRB host galaxies. Levesque et al. (2010) (see also Han et al. 2010) showed that there is an offset between the $M-Z$ relation of LGRB hosts with $z<0.3$ and that of nearby SDSS star-forming galaxies and between DEEP2 emission-line galaxies at $\langle z\rangle=0.8$ and LGRB hosts with $0.3<z<1$. These works indicate that LGRBs occur preferentially in galaxies with lower metallicity than the general star-forming galaxy population. On the other hand, they also show that LGRB host galaxies do not follow a metallicity cut-off and therefore a low-metallicity host does not seem to be a key driver of the production of a LGRB. This result can also be extended to the LGRB environment, assuming that for these low-mass galaxies there is a small metallicity gradient and that the average metallicity of the host is comparable to that of the GRB environment (e.g. Levesque et al. 2011).

Mannucci et al. (2010) found a new general relation between the stellar mass, the metallicity, and the SFR of galaxies (see also Lara-López et al. 2010). This so-called fundamental metallicity relation (FMR) implies that, for a given $M_{\star}$, galaxies with higher SFR have lower metallicities and is explained by the authors by a complex interplay between infall gas dilution, SFR timescale, and gas outflows. Mannucci et al. (2011) use the FMR to investigate the $M-Z$ relation of LGRB hosts and show that they follow the extrapolation of the FMR to low masses (see however Kocevski \& West 2011). This suggests that a key property of LGRB hosts could be the SSFR. There is indeed a cut-off at $\log (S S F R) \geq-10$ in the SSFR of LGRB hosts, indicating a high efficiency in forming stars.

The host of GRB 091127 extends to lower stellar masses the sample of LGRB host galaxies at $0.3<z<1$. The $M_{\star}$ and metallicity values we find broaden the space covered by the LGRB hosts in the $M-Z$ plot presented by Levesque et al. (2010), but can still be consistent with a $M-Z$ shift of LGRB hosts compared with the $M-Z$ relation found from emission-line galaxy surveys. The SSFR value fulfills the above-mentioned cut-off and the $M_{\star}$, metallicity, and SFR of the host of GRB 091127 are in perfect agreement with the extended-FMR relation (see Fig. 6). Moreover, we can use the FMR to predict the host metallicity starting from the $M_{\star}$ and SFR values determined. We use Eq. (2) of Mannucci et al. (2011) and obtain $12+\log (\mathrm{O} / \mathrm{H})=8.3$, in perfect agreement with our determined value. This exercise is an example of the potential of the FMR: if this relation is confirmed, it will be possible to determine all the three properties of galaxies even if the available observations are useful for determining only two of them.

We do not detect any Wolf-Rayet emission lines/bumps. Even though the detection of these features are expected if WolfRayet stars are the progenitors of LGRBs, the signal-to-noise ratios and flux limits of our spectra make the detection impossible for lines with similar luminosities as those found by Han et al. (2010).

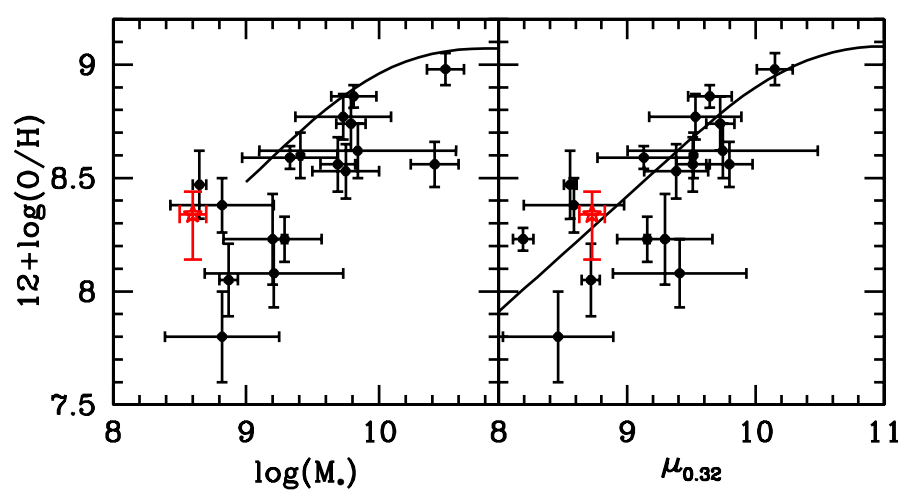

Fig. 6. Left panel: $M-Z$ realtion. The black points with error bars correspond to the GRB $M_{\star}$ and metallicities used in Mannucci et al. (2011), whereas the black line shows the fit to the local $M-Z$ relation presented in the same paper. Right panel: FMR relation. The black points with error bars correspond to the GRB $\mu_{0.32} \equiv \log \left(M_{\star}\right)-0.32 \log (S F R)$ and metallicities used in Mannucci et al. (2011), whereas the black line shows the extended FMR relation presented in the same paper. In both panels, the values for GRB 091127 are represented by a red star with error bars. A colour version of this figure is available online.

The $2 \mathrm{D}$ velocity and velocity dispersion maps show that the kinematics of the GRB 091127 host is complex. Following the works of Flores et al. (2006) and Puech et al. (2006) for a sample of galaxies at similar redshift studied with VLT/GIRAFFE, the perturbed kinematics could be associated with the presence of strong inflows/outflows or mergers (ongoing or remnant). Nonetheless, it should be noted that the galaxies analysed by these authors are much more massive and luminous than LGRB hosts.

The afterglow position is consistent with the region of the host characterised by a high SFR, as expected from the findings of Fruchter et al. (2006). The peak of the SFR map is close to the position of the velocity dispersion maximum. Green et al. (2010) using high resolution $3 \mathrm{D}$ observations, found that the high velocity dispersion is correlated with the star formation rates, claiming that star formation itself is the energetic driver of galaxy disk turbulence. Numerical simulations have already shown the same effect. In our case, we are studying coarse maps and we only have information on a large scale. From our analysis, it is impossible to assess a relation between the excess of SFR and the velocity dispersion behavior. Further modeling is necessary and a detailed analysis in this sense will be presented in a future project dedicated to the X-shooter GTO IFU LGRB host galaxy survey.

\section{Conclusions}

We have analysed the afterglow of GRB 091127 using both Swift-XRT and ground-based data. While the delay in the $\mathrm{X}$-ray observations prevents us from studying the prompt/early emission characteristics as done in Starling et al. (2011), we have confirmed the presence of a SN bump as reported by Cobb et al. (2010) (the GRB091127/SN 2009nz association was also claimed spectroscopically by Berger et al. 2011) and we point out the presence of a possible jet break, showing that GRB 091127 follows the so-called Ghirlanda relation.

We have carried out a detailed study of the properties of the host galaxy at $z \sim 0.49$, using both slit and IFU X-shooter echelle spectroscopy. 2D GRB host maps have been previously produced only for the host of GRB 980425 in the local universe (Christensen et al. 2008). 
Besides showing that the GRB 091127 host has similar integrated properties as LGRB hosts in general, our results extend to lower values the $M_{\star}$ derived for the sample of LGRB hosts at $0.3<z<1.0$, hence adding new information to explore the faint end of the $M-Z$ relation. To obtain a conclusive picture of the shift in the LGRB host $M-Z$ relation from that found from emission-line galaxy surveys, it is necessary to increase the number of detailed studies of LGRB hosts. The SFR, $M_{\star}$, and $Z$ values determined are in perfect agreement with the FMR extension to low masses.

We stress the importance of LGRB hosts in studying the properties of low-mass galaxies, since these galaxies are very often underrepresented in current surveys. The $M-Z$ is a fundamental relation for understanding galaxy evolution. It is therefore very important to enlarge the sample of LGRB hosts to check wheter the $M-Z$ offset is confirmed, to see how it evolves with redshift, and to test the FMR, with the main purpose of understanding the processes that produce the $M-Z$ relation. Thanks to the X-shooter sensitivity and wavelength coverage, our GTO program dedicated to GRB host spectroscopy will enable us to significantly enlarge the sample of studied LGRB host galaxies and extend it to higher redshift.

IFU observations add detailed information to study this class of objects, which are not selected primarily on their luminosity and therefore form a complementary sample to those of current surveys of galaxies. 2D maps can also be used to understand which factors are required to drive a GRB explosion, and, for the cases with suitable spatial resolution, to determine the properties of the GRB region. The X-shooter IFU observation of the GRB 091127 host galaxy indicates a galaxy with perturbed rotation and a SFR enhancement towards the centre of the host. The connection between turbulence and SFR remains disputed. Thanks to the IFU X-shooter GTO LGRB host galaxy survey that we are carrying out within the French-Italian X-shooter GTO collaboration, it will be possible to produce systematically $2 \mathrm{D}$ velocity, SFR, and also metallicity and electron density maps for more than 15 LGRB host galaxies at $z<0.6$. This study will provide a much more detailed knowledge of the properties of these galaxies and of the GRB environment, to be compared with those of the star-forming galaxy population and in particular with the host galaxies of broad-lined Ic SNe.

Acknowledgements. This work is partially based on observations with: Gran Telescopio Canarias (GTC), instaled in the Spanish Observatorio del Roque de los Muchachos of the Instituto de Astrofísica de Canarias in the island of La Palma; the Centro Astronòmico Hispano Alemán (CAHA) at Calar Alto, operated jointly by the Max-Planck Institut für Astronomie and the Instituto de Astrofísica de Andalucía (IAA-CSIC); the Nordic Optical Telescope, operated on the island of La Palma jointly by Denmark, Finland, Iceland, Norway, and Sweden, in the Spanish Observatorio del Roque de los Muchachos of the Instituto de Astrofísica de Canarias; the APEX telescope under the ESO program E-084.D-0732A. This work made use of data supplied by the UK Swift Science Data Centre at the University of Leicester. We thank the referee, Emily Levesque, for the accurate review of the paper. S.D.V. acknowledges useful correspondence with F. Mannucci, M. Modjaz and L. Nava. S.D.V. thanks S. Savaglio and T. Vinci for precious help. J.G., R.S., A.J.C.T., J.C.T., M.J., acknowledge support by the Spanish Ministry of Science and Innovation (MICINN) under the project grants AYA2008-03467/ESP and AYA2009-14000C03-01 (including Feder funds). P.J. acknowledges support by a Marie Curie European Reintegration Grant within the 7th European Community Framework Program, and a Grant of Excellence from the Icelandic Research Fund. The Dark Cosmology Centre is funded by the Danish National Research Foundation.

\section{References}

Amati, L. 2006, MNRAS, 372, 233

Baldry, I. K., \& Glazebrook, K. 2003, ApJ, 593, 258

Berger, E., Chornock, R., Holmes, T. R., et al. 2011, ApJ, submitted [arXiv: 1106.3073$]$

Bloom, J. S., Kulkarni, S. R., Djorgovski, S. G., et al. 1999, Nature, 401, 453
Bloom, J. S., Kulkarni, S. R., \& Djorgovski, S. G. 2002, AJ, 123, 111 Bruzual, G. 2007, From Stars to Galaxies: Building the Pieces to Build Up the Universe, ed. A. Vallenari, R. Tantalo, L. Portinari, \& A. Moretti, ASP Conf. Ser., 374, 303

Bruzual, G., \& Charlot, S. 2003, MNRAS, 344, 1000

Calzetti, D., Kinney, A. L., \& Storchi-Bergmann, T. 1994, ApJ, 429, 582

Castro-Tirado, A. J., Møller, P., García-Segura, G., et al. 2010, A\&A, 517, A61 Chabrier, G. 2003, PASP, 115, 763

Chen, H.-W., Prochaska, J. X., Ramirez-Ruiz, E., et al. 2007, ApJ, 663, 420 Christensen, L., Vreeswijk, P. M., Sollerman, J., et al. 2008, A\&A, 490, 45 Cobb, B. E., Bloom, J. S., Perley, D. A., et al. 2010, ApJ, 718, L150

Covino, S., Campana, S., Conciatore, M. L., et al. 2010, A\&A, 521, A53

Crowther, P. A. 2007, ARA\&A, 45, 177

Cucchiara, A., Fox, D., Levan, A., \& Tanvir, N. 2009, GCN, 10202

Dahlen, T., Mobasher, B., Somerville, R. S., et al. 2005, ApJ, 631, 126

Della Valle, M., Malesani, D., Benetti, S., et al. 2003, A\&A, 406, L33

Della Valle, M., Chincarini, G., Panagia, N., et al. 2006, Nature, 444, 1050

di Paola, A. 2001, in Gamma-ray Bursts in the Afterglow Era, ed. E. Costa, F. Frontera, \& J. Hjorth, 390

D’Odorico, S., Andersen, M. I., Conconi, P., et al. 2004, in SPIE Conf. Ser. 5492, ed. A. F. M. Moorwood, \& M. Iye, 220

Evans, P. A., Beardmore, A. P., Page, K. L., et al. 2007, A\&A, 469, 379

Evans, P. A., Beardmore, A. P., Page, K. L., et al. 2009, MNRAS, 397, 1177

Fiore, F., D'Elia, V., Lazzati, D., et al. 2005, ApJ, 624, 853

Flores, H., Hammer, F., Puech, M., Amram, P., \& Balkowski, C. 2006, A\&A, 455,107

Flores, H., Goldoni, P., Royer, F., et al. 2011, Astron. Nachr., 332, 288

Fruchter, A. S., Levan, A. J., Strolger, L., et al. 2006, Nature, 441, 463 Fynbo, J. P. U., Watson, D., Thöne, C. C., et al. 2006, Nature, 444, 1047 Gal-Yam, A., Fox, D. B., Price, P. A., et al. 2006, Nature, 444, 1053

Galama, T. J., Groot, P. J., van Paradijs, J., et al. 1998, ApJ, 497, L13

Galama, T. J., Tanvir, N., Vreeswijk, P. M., et al. 2000, ApJ, 536, 185

Ghirlanda, G., Ghisellini, G., \& Lazzati, D. 2004, ApJ, 616, 331

Ghirlanda, G., Ghisellini, G., \& Firmani, C. 2005, MNRAS, 361, L10

Goldoni, P., Royer, F., François, P., et al. 2006, in SPIE Conf. Ser., 6269

Granot, J. 2007, in Rev. Mex. Astron. Astrofis. Conf. Ser., 27, 140

Green, A. W., Glazebrook, K., McGregor, P. J., et al. 2010, Nature, 467, 684

Guetta, D., \& Della Valle, M. 2007, ApJ, 657, L73

Hammer, F., Flores, H., Schaerer, D., et al. 2006, A\&A, 454, 103

Han, X. H., Hammer, F., Liang, Y. C., et al. 2010, A\&A, 514, A24

Heger, A., Fryer, C. L., Woosley, S. E., Langer, N., \& Hartmann, D. H. 2003, ApJ, 591, 288

Hjorth, J., \& Bloom, J. S. 2011, Chapter 9 in Gamma-Ray Bursts, ed. C. Kouveliotou, R. A. M. J. Wijers, \& S. E. Woosley (Cambridge University Press) [arXiv: 1104.2274]

Kelly, P. L., Kirshner, R. P., \& Pahre, M. 2008, ApJ, 687, 1201

Kennicutt, Jr., R. C. 1998, ARA\&A, 36, 189

Kewley, L. J., \& Ellison, S. L. 2008, ApJ, 681, 1183

Kobulnicky, H. A., \& Kewley, L. J. 2004, ApJ, 617, 240

Kocevski, D., \& West, A. A. 2011, ApJ, 735, L8

Lara-López, M. A., Cepa, J., Bongiovanni, A., et al. 2010, A\&A, 521, L53

Lazzati, D., Covino, S., Ghisellini, G., et al. 2001, A\&A, 378, 996

Levesque, E. M., Kewley, L. J., Berger, E., \& Jabran Zahid, H. 2010, AJ, 140 1557

Levesque, E. M., Berger, E., Soderberg, A. M., \& Chornock, R. 2011, ApJ, 739, 23

Liang, E.-W., Zhang, B.-B., \& Zhang, B. 2007, ApJ, 670, 565

MacFadyen, A. I., \& Woosley, S. E. 1999, ApJ, 524, 262

Maiolino, R., Nagao, T., Grazian, A., et al. 2008, A\&A, 488, 463

Mannucci, F., Cresci, G., Maiolino, R., Marconi, A., \& Gnerucci, A. 2010, MNRAS, 408, 2115

Mannucci, F., Salvaterra, R., \& Campisi, M. A. 2011, MNRAS, 439

McKenzie, E. H., \& Schaefer, B. E. 1999, PASP, 111, 964

Meliani, Z., \& Keppens, R. 2010, A\&A, 520, L3

Mirabal, N., Halpern, J. P., Chornock, R., et al. 2003, ApJ, 595, 935

Modjaz, M., Kewley, L., Kirshner, R. P., et al. 2008, AJ, 135, 1136

Morrison, R., \& McCammon, D. 1983, ApJ, 270, 119

Nava, L., Ghirlanda, G., Ghisellini, G., \& Celotti, A. 2011, A\&A, 530, A21

Osterbrock, D. E. 1989, Astrophysics of gaseous nebulae and active galactic nuclei (Mill Valley, CA: University Science Books)

Panaitescu, A., Mészáros, P., Gehrels, N., Burrows, D., \& Nousek, J. 2006, MNRAS, 366, 1357

Patat, F., Cappellaro, E., Danziger, J., et al. 2001, ApJ, 555, 900

Pei, Y. C. 1992, ApJ, 395, 130

Pettini, M., \& Pagel, B. E. J. 2004, MNRAS, 348, L59

Puech, M., Hammer, F., Flores, H., Östlin, G., \& Marquart, T. 2006, A\&A, 455, 119

Salpeter, E. E. 1955, ApJ, 121, 161 
Savaglio, S., Glazebrook, K., \& Le Borgne, D. 2009, ApJ, 691, 182 Schaefer, B. E., Gerardy, C. L., Höflich, P., et al. 2003, ApJ, 588, 387 Schlegel, D. J., Finkbeiner, D. P., \& Davis, M. 1998, ApJ, 500, 525 Skrutskie, M. F., Cutri, R. M., Stiening, R., et al. 2006, AJ, 131, 1163 Soderberg, A. M., Chakraborti, S., Pignata, G., et al. 2010, Nature, 463, 513 Sollerman, J., Kozma, C., Fransson, C., et al. 2000, ApJ, 537, L127

Sollerman, J., Holland, S. T., Challis, P., et al. 2002, A\&A, 386, 944 Sollerman, J., Östlin, G., Fynbo, J. P. U., et al. 2005, New Astron., 11, 103 Sparre, M., Sollerman, J., Fynbo, J. P. U., et al. 2011, ApJ, 735, L24

Starling, R. L. C., Wijers, R. A. M. J., Hughes, M. A., et al. 2005, MNRAS, 360, 305

Starling, R. L. C., Wiersema, K., Levan, A. J., et al. 2011, MNRAS, 411, 2792
Thöne, C. C., Fynbo, J. P. U., Östlin, G., et al. 2008, ApJ, 676, 1151 Thöne, C. C., Goldoni, P., Covino, S., et al. 2009, GCN, 10233, 233, 1 van Dokkum, P. G. 2001, PASP, 113, 1420

van Eerten, H. J., \& MacFadyen, A. I. 2011 [arXiv: 1105.2485] van Marle, A. J., Langer, N., Yoon, S.-C., \& García-Segura, G. 2008, A\&A, 478, 769

Wilson-Hodge, C. A., \& Preece, R. D. 2009, GCN, 10204, 204, 1 Woosley, S. E. 1993, ApJ, 405, 273

Yang, Y., Flores, H., Hammer, F., et al. 2008, A\&A, 477, 789

Yoon, S.-C., Woosley, S. E., \& Langer, N. 2010, ApJ, 725, 940

Zhang, B., \& Mészáros, P. 2004, Int. Mod. Phys. A, 19, 2385

Zhang, B., Fan, Y. Z., Dyks, J., et al. 2006, ApJ, 642, 354 